\title{
O uso da prosódia na sinalização da estrutura do discurso em Kisedjê
}

\author{
Miguel Oliveira, JR.
}

Professor de Linguística na Universidade Federal de Alagoas (UFAL).

Resumo: 0 presente trabalho tem por objetivo investigar se - tal como acontece em diversas línguas amplamente documentadas e analisadas - a prosódia participa na sinalização da estrutura do discurso em Kisêdjê, uma língua nativa brasileira pertencente ao grupo Jê. Para esse fim, os seguintes elementos prosódicos foram selecionados para análise: a pausa, a diferença de tom e 0 tom de fronteira.

Palavras-chave: Prosódia; Discurso; Língua indígena brasileira; Kisêdjê
Abstract: The present study investigates whether - as in several well-documented languages -prosody plays a role in the signaling of discourse segmentation in Kisêdjê, a Brazilian native language of the Jê group. Inspired by the literature, the following prosodic variables were selected for analysis: pause, pitch reset and boundary tones.

Keywords: Prosody; Discourse; Brasilian native language; Kisêdjê 



\section{Introdução}

Qualquer tipo de discurso é formado por conjuntos de frases que possuem uma relação semântica coerente entre si. A estrutura do discurso escrito é o mais das vezes clara, por conta do uso de convenções tipográficas, tais como a pontuação e a organização do texto ${ }^{1}$ em parágrafos. O discurso falado faz uso de outros mecanismos para sinalizar sua estrutura.

Estudos recentes na área da linguística computacional têm mostrado que a prosódia é usada para delimitar estruturas discursivas que constituem macro-unidades coerentes (GELUYKENS \& SWERTS, 1994; GROSZ \& HIRSCHBERG, 1992; PASSONNEAU \& LITMAN, 1993). De acordo com tais estudos, as unidades discursivas da fala são geralmente separadas por meio de elementos prosódicos, tais como a pausa, a entoação e a velocidade da fala. $\mathrm{O}$ uso de tais elementos prosódicos contribui para a identificação semântica das unidades discursivas, assim como explicita quais as intenções do falante para com sua audiência. Isso claramente facilita o processo da comunicação.

Independentemente de evidências prosódicas, algumas modalidades discursivas têm uma estrutura interna que pode ser verificada apenas se se levar em consideração o conteúdo de seus constituintes. Textos procedimentais, por exemplo, são compostos por segmentos (seções ou unidades) semanticamente independentes que são facilmente reconhecíveis por conta do encadeamento causal que apresentam. Para Aouladomar \& Saint-Dizier (2005) e Aouladomar (2005), os textos procedimentais são, em geral, altamente estruturados e exibem um arcabouço microrretórico particularmente rico que está integrado nas estruturas sintático-semânticas das instruções e das ações preparatórias. Uma vez que a prosódia é regularmente utilizada para segmentar "blocos de informações" no discurso, como se tem amplamente demonstrado, deduzse que os segmentos semanticamente independentes em
${ }^{1}$ No presente trabalho, as palavras discurso e texto são usadas comutativamente, sem qualquer diferença de significado. 
textos procedimentais são também delimitados por elementos prosódicos, o que deixa transparecer a sua estrutura.

A língua Kisêdjê, pertencente à família linguística Jê, que, por sua vez, pertence ao tronco linguístico Macro-Jê, é falada por cerca de 370 pessoas dentro e nas imediações do Parque Indígena do Xingu, no Mato Grosso (Brasil), sendo, portanto, um língua ameaçada de extinção. Existem dois subgrupos da família Jê: o Jê Central e o Jê do Noroeste. A língua Kisêdjê pertence ao grupo Jê do Noroeste, que também inclui a Apinayé, a Kayapó, a Kreen Akarore, e a Timbira. Kisêdjê é uma das línguas Jê menos estudadas. (RODRIGUES, 1999). O povo Kisêdjê é o único do grupo Jê que habita o Parque Indígena do Xingu. O primeiro contato registrado dos Kisêdjê com não indígenas se deu em 1884, durante a expedição de Karl von den Steinen, médico, explorador, etnólogo e antropólogo alemão, que, com o apoio de entidades alemãs e brasileiras, promoveu diversas expedições na região central do Brasil. O contato com não indígenas se tornaria mais intenso para os Kisêdjê depois da intervenção dos irmãos Vilas Boas, que, em 1959, propuseram um acordo de pacificação e de deslocamento dentro do Parque Indígena. No entanto, a despeito desse contato, e da adoção de alguns costumes e rituais do povo xinguano, os Kisêdjê sempre permaneceram fieis à sua cultura e à sua língua. São poucos os Kisêdjê que dominam a lingua franca da região (o português), sendo a maioria dessas pessoas homens mais velhos, que participaram de algum modo da intensa negociação promovida na década de 60 pelos irmãos Vilas Boas. No geral, mulheres, crianças e adolescentes falam exclusivamente Kisêdjê.

\section{Metodologia}

O material utilizado neste estudo é composto por quatro textos procedimentais proferidos por duas falantes de Kisêdjê. Os textos PC_050326_083 e $P C \_050326 \_09$ foram produzidos por uma mulher de 55 
anos de idade e versam sobre o preparo do beiju (pão de mandioca) e da perereba (uma bebida feita de mandioca doce), respectivamente. Os textos PC_050403_05 e $P C \_050403 \_06$ foram produzidos por uma mulher de 27 anos de idade e versam sobre a feitura da esteira de talo (uma espécie de peneira usada para, entre outras coisas, coar a mandioca processada) e do $k h i$ (batata cozida). Os textos, de duração variada (de $50 \mathrm{seg}$. a $1 \mathrm{~min} .54 \mathrm{seg}$.), perfazem um total de $5 \mathrm{~min}$. $21 \mathrm{seg}$. de gravação. As gravações foram feitas com um microfone unidirecional auricular Shure conectado a um gravador DAT TASCAM. Um falante nativo da língua, que é professor na aldeia principal do povo Kisêdjê, foi responsável pela tradução dos textos para o português.

Para minimizar o risco de circularidade subjacente aos estudos que investigam o papel da prosódia na estruturação do discurso, a literatura especializada tem proposto que se utilize um modelo discursivo independente. (BROWN, CURRIE \& KENWORTHY, 1980; SWERTS, 1997). Vários estudos feitos para diversas línguas têm evidenciado que não existe uma correspondência direta entre a organização sintática do discurso e a estrutura prosódica a ele associada. (CUTLER, DAHAN \& DONSELAAR, 1994; PIJPER \& SANDERMAN, 1994). O que esses estudos têm demonstrado é que a utilização de elementos prosódicos no discurso está muito mais relacionada a aspectos semânticos e pragmáticos do discurso que à sua disposição sintática. Portanto, não é recomendável que a análise apoie-se na sintaxe, o que seria a opção mais imediata.

A fim de trabalhar com um modelo de estrutura de informação contra a qual a prosódia pode ser confrontada, alguns autores apoiam-se em segmentações propostas por diversas linhas da análise do discurso. (GROSZ \& HIRSCHBERG, 1992; GROSZ \& SIDNER, 1986; HIRSCHBERG \& GROSZ, 1992; LITMAN \& PASSONNEAU, 1995; NAKATANI \& HIRSCHBERG, 1995; PASSONNEAU \& LITMAN, 1993). O problema com 
o uso da abordagem da análise de discurso é que, a priori, não se sabe se o resultado ultrapassará a mera intuição que se tem acerca da estrutura do discurso. Se se opta por utilizar um método independente de segmentação do discurso, é preciso garantir que se empregue um modelo replicável, uma vez que quanto maior a reprodutibilidade de um modelo de segmentação do discurso, mais forte será a evidência de que uma estrutura subjacente ao discurso de fato existe.

No presente estudo, utilizou-se o modelo proposto por Passonneau \& Litman (1993), que produz uma segmentação linear baseada na intenção do falante. Este modelo tem sido amplamente usado em estudos de prosódia em diversas línguas; o seu emprego aqui possibilitará, portanto, eventuais comparações com resultados obtidos em trabalhos realizados para outras línguas. Além disso, estudos recentes têm demonstrado que este modelo apresenta um nível razoável de consenso entre codificadores, o que o torna mais fiável e reproduzível. (ver ARIM, COSTA \& FREITAS, 2003, para mais detalhes).

Seguindo os procedimentos adotados em Passonneau \& Litman (1993), oito codificadores leigos participaram de um teste preambular para verificar a replicabilidade do modelo proposto pelas autoras. Os codificadores foram solicitados a segmentar o material de estudo levando em consideração a intenção do ouvinte como critério. Foi observado um alto valor de consenso entre codificadores (kappa > 0.7), o que validou também aqui o emprego do modelo em questão.

Todos os quatro textos procedimentais foram inicialmente divididos em unidades de tom e, posteriormente, segmentados em blocos sequenciais, cada um representando uma única intenção, conforme proposto pelo modelo de Passonneau \& Litman (1993). Essa segmentação foi feita tendo por base a segmentação proposta pelos codificadores leigos. Unidade de tom aqui, como nos trabalhos de Cruttenden (1997) e de Crystal 
(1969), foi, para efeito de análise, considerada uma unidade acústica composta de pelo menos uma sílaba proeminente e um movimento tonal destacado (o núcleo). A identificação de fronteiras de unidades de tom foi, dessa maneira, feita a priori, levando-se em conta pistas fonéticas. Em alguns casos mais complexos, características semânticas e gramaticais foram também consideradas. A divisão dos textos em blocos sequenciais (ou unidades discursivas) levou em consideração a segmentação previamente feita dos textos em unidades de tom, de maneira que as fronteiras discursivas necessariamente coincidiram com fronteiras de unidades de tom. Os quatro textos contêm, em conjunto, um total de 215 unidades de tom, 38 das quais foram identificadas como fronteira de uma unidade discursiva. Para cada unidade de tom, foram designados um núcleo e o tipo de tom de fronteira (baixo ou não-baixo). A opção por identificar o tom de fronteira como baixo ou não-baixo foi inspirada por problemas discutidos na literatura acerca da imprecisão na distinção entre os tons "alto" e "médio". (BROWN, CURRIE, \& KENWORTHY, 1980; GELUYKENS \& SWERTS, 1994; SWERTS, 1997; VAN DONZEL, 1999).

Três elementos prosódicos foram escolhidos para análise: a pausa, o tom de fronteira e a diferença de tom. Seguindo os trabalhos de Henderson, Goldman-Eisler \& Skarbeck (1965) e de Hieke, Kowal \& O'Connell (1983), considerou-se pausa aqui como um período de silêncio igual ou superior a $100 \mathrm{~ms}$. A diferença de tom foi calculada como a diferença entre os valores da extensão de tom entre duas unidades de tom adjacentes. A medida de extensão de tom aqui correspondeu ao valor da frequência fundamental máxima na unidade de tom. Esse valor foi auferido na vogal da sílaba da unidade de tom em que se detectava o pico da frequência fundamental (GROSZ \& HIRSCHBERG, 1992; HIRSCHBERG \& GROSZ, 1992; MENN \& BOYCE, 1982; NAKATANI \& HIRSCHBERG, 1995).

Os valores de tom foram computados automaticamente, 
usando o algoritmo padrão de extração de frequência fundamental do programa Praat (BOERSMA, 2005). As curvas de tom originais foram manualmente estilizadas, num processo semi-automático, levando em conta características auditivas e visuais dos documentos de áudio. Esse procedimento foi feito para evitar a interferência de pulos de oitava e para suavizar as curvas de tom, tornando-as assim mais facilmente analisáveis (NOOTEBOOM \& KRUYT, 1987; VAN DONZEL, 1999). Os valores de frequência fundamental, expressos em semitons, foram arredondados no cômputo final.

\section{Hipóteses}

A hipótese central do presente trabalho é de que as fronteiras de unidades discursivas são prosodicamente diferentes das fronteiras de unidades de tom que ocorrem no interior de unidades discursivas. Isso poderá ser verificado, especificamente, através dos seguintes elementos prosódicos:

- Pausa: pausas mais longas tendem a ocorrer com mais frequência nas fronteiras discursivas que em outros pontos no texto;

- Diferença de tom: a diferença de tom é maior nas fronteiras discursivas que em outros pontos no texto, e

- Tom de fronteira: o tom baixo tende a ocorrer no final das unidades discursivas, enquanto que o tom não-baixo geralmente se encontra dentro das unidades discursivas.

A hipótese, assim, relaciona três variáveis prosódicas a diferentes tipos de fronteiras em textos procedimentais: fronteiras que coincidem com o fim de unidades discursivas e fronteiras que ocorrem dentro dessas unidades. Apoia-se em estudos correlatos acerca da prosódia como marca de estruturação do discurso (cf. referências na Introdução). 


\section{Resultados}

\subsection{Pausa}

Diversos estudos já demonstraram que a pausa é utilizada sistematicamente para demarcar fronteiras de unidades de tom (BROWN, CURRIE \& KENWORTHY, 1980; Chafe, 1989). O uso da pausa no discurso tem efeitos muito mais abrangentes: num nível global, a pausa assume uma função mais específica na organização de textos.

A literatura tem demonstrado uma relação significativa entre a duração da pausa e a posição em que ocorrem no discurso: quanto mais longa a pausa, maiores as chances de o local em que ocorre coincidir com uma ruptura discursiva (ver, por exemplo, GEE \& GROSJEAN, 1984; GROSZ \& HIRSCHBERG, 1992; KOWAL \& O'CONNELL, 1987; PASSONNEAU \& LITMAN, 1993, entre outros). Em Kisêdjê, este também parece ser o caso, conforme tabela 1 abaixo sugere:

Tabela 1: Duração média da pausa em fronteiras de unidades discursivas (SIM) e em outros lugares (NÃO), expressa em milissegundos.

\begin{tabular}{|l|l|}
\hline $\begin{array}{l}\text { Fronteira } \\
\text { Discursiva }\end{array}$ & $\begin{array}{l}\text { Duração } \\
\text { da Pausa }\end{array}$ \\
\hline SIM & $871 \mathrm{mseg}$. \\
NÃO & $641 \mathrm{mseg}$. \\
\hline
\end{tabular}

A duração média das pausas que ocorrem em fronteiras de unidades discursivas são significativamente diferentes da duração média daquelas que ocorrem em outros lugares. Resultados de testes estatísticos $(t=-2,909$, $\mathrm{df}=113, \mathrm{p}<0,005)$ confirmam esta observação, mostrando uma correlação significativa entre tipos de fronteira (discursiva ou não) e duração da pausa.

\subsection{Tom de fronteira}

Os movimentos tonais associados ao fim de uma unidade de tom têm sido sistematicamente estudados 
em trabalhos que investigam a relação entre a prosódia e a segmentação do discurso. Em geral, argumenta-se que os contornos melódicos promovem a distinção entre continuidade e finalização, sendo os tons alto e médio geralmente associados à noção de continuidade e o tom baixo associado à noção de finalização. (BLAAUW, 1995; BROWN, CURRIE \& KENWORTHY, 1980; GELUYKENS \& SWERTS, 1994; VAN DONZEL, 1999; WICHMAN, HOUSE \& RIETVELD, 1997). Os resultados obtidos no presente estudo sugerem que a mesma estratégia é empregada em Kisêdjê. A tabela 2 abaixo mostra a diferença (em porcentagem) na distribuição de tons de fronteira entre fronteiras discursivas (SIM) e fronteiras encontradas no interior de unidades discursivas (NÃO). A partir desses resultados, pode-se deduzir que as unidades de tom que finalizam uma unidade discursiva são, na maioria das vezes ( $66 \%$ dos casos), associadas a um tom de fronteira baixo; as unidades de tom que, por outro lado, encontram-se a meio de uma unidade discursiva, vêm em geral acompanhadas por um tom de fronteira não-baixo (75\% de todos os casos). Essa diferença é estatisticamente significativa $(\mathrm{X} 2=21,810, \mathrm{df}=213, \mathrm{p}<0,001)$.

Tabela 2: Distribuição de tom de limite (baixo e não-baixo) em função dos tipos de fronteira (no final de uma unidade discursiva (SIM) e em outro lugar, (NÃO). Os valores são em relação à quantidade total de ocorrências.

\begin{tabular}{|l|l|l|}
\hline Fronteira & \multicolumn{2}{|l|}{ Tom de Fronteira } \\
\hline Discursiva & Baixo & Não-Baixo \\
\hline SIM & $66 \%$ & $34 \%$ \\
NÃO & $25 \%$ & $75 \%$ \\
\hline
\end{tabular}

\subsection{Diferença de tom}

A literatura tem sugerido que a descontinuidade melódica que ocorre entre unidades de informação - uma consequência do declínio natural do tom no curso de um enunciado - funciona como uma importante pista para a estruturação do discurso (GROSZ \& HIRSCHBERG, 
1992; HAKODA \& SATO, 1980; HIRSCHBERG \& GROSZ, 1992; NAKAJIMA \& ALLEN, 1992; PIJPER \& SANDERMAN, 1994; SWERTS, 1997). O quadro 3 abaixo indica uma associação muito clara entre o valor da diferença de tom e o tipo de fronteira (discursiva ou não) em Kisêdjê:

Tabela 3: Valores médios de diferença de tom no final de uma unidade de discurso (SIM) e em outros lugares (NÃO). Os valores estão expressos em semitons.

\begin{tabular}{|l|l|}
\hline $\begin{array}{l}\text { Fronteira } \\
\text { Discursiva }\end{array}$ & $\begin{array}{l}\text { Diferença de Tom } \\
\text { (em semitons) }\end{array}$ \\
\hline SIM & 3.26 \\
NÃO & 1.98 \\
\hline
\end{tabular}

Os valores mais elevados de diferença de tom estão claramente associados às fronteiras discursivas, como demonstra a tabela acima. As análises estatísticas oferecem resultados significativos $(t=-2,975$, $d f=213$, $\mathrm{p}<0,0032$ ). Portanto, a diferença de tom parece ser um indicador muito preciso de fronteiras discursivas em textos procedimentais em Kisêdjê.

\section{Discussão}

O presente trabalho teve por objetivo investigar se a língua nativa brasileira Kisêdjê, pertencente ao grupo linguístico Jê, faz uso da prosódia como um dispositivo de estruturação do discurso, tal como acontece em diversas línguas europeias. Para isso, utilizou-se aqui um modelo empírico baseado na intenção do falante, com o objetivo de verificar se determinadas variáveis prosódicas (o tom de fronteira, a pausa, e a diferença de tom) estão sistematicamente correlacionadas à estrutura interna de um tipo particular de texto: o discurso procedimental.

A análise dos dados mostrou que a pausa é significativamente utilizada em Kisêdjê como um meio de segmentação de textos procedimentais em 
blocos de informação. Especificamente, as pausas são consistentemente mais longas no final de unidades discursivas que no interior dessas unidades, sugerindo assim que essa característica prosódica é um recurso comum na tarefa de fazer com que a estrutura de textos procedimentais, segmentados em seções caracterizadas por uma coerência semântica, torne-se transparente para o ouvinte.

Verificou-se também que a diferença de valores de extensão de tom entre unidades de tom adjacentes constitui uma sinalização relevante da segmentação do discurso na língua - unidades discursivas tendem a ser separadas uma das outras por meio de um valor mais alto de diferença de tom.

Além disso, a maneira como a curva melódica associada à unidade de tom finaliza a sua trajetória também fornece indícios acerca da estruturação de textos procedimentais em Kisêdjê, uma vez que indica com regularidade se uma dada unidade discursiva chegou ao fim ou não. Curvas melódicas que finalizam com um tom baixo costumam indicar o fim de uma unidade discursiva nos textos aqui analisados.

Evidentemente, estas conclusões nada oferecem de novo no que diz respeito ao estudo das características prosódicas no discurso, mas são, no entanto, de interesse por pelo menos uma razão bastante importante: muito pouco ainda se sabe sobre a organização do discurso em línguas nativas brasileiras, em especial sobre se a prosódia é empregada como um mecanismo de estruturação em quaisquer dessas línguas - algo que acontece em várias línguas europeias, como demonstra fartamente a literatura. Seria, obviamente, ingênuo supor que a língua Kisêdjê apresentaria as mesma características de línguas europeias ou usaria os mesmos tipos de estratégia prosódicas empregadas por línguas já bem documentadas na organização estrutural do discurso. Desse modo, este estudo preliminar constitui uma necessária contribuição para o debate. Afinal, só com um número significativo 
de pesquisas sobre diferentes aspectos da prosódia do discurso, realizados em diferentes comunidades, podese testar a validade dos resultados existentes. Isso, consequentemente, proporcionará uma compreensão mais profunda dos diversos mecanismos de discurso presentes em diferentes línguas e culturas.

\section{Conclusão}

$\mathrm{O}$ presente estudo experimental demonstrou que falantes de Kisêdjê fazem uso sistemático da prosódia como um recurso de estruturação do discurso, segmentando blocos de informações com pausas mais longas, tom de limite baixo e elevadas diferenças de tom. Isto sugere que eles parecem estar cientes da existência de uma estrutura subjacente ao discurso - no caso, ao discurso procedimental -, algo que é evidenciado através do uso regular dos recursos prosódicos mencionados. Outras características prosódicas, tais como a intensidade, o alongamento da vogal final, e a velocidade de fala também devem ser estudadas no futuro. A prosódia, no entanto, não é o único mecanismo que pode ser utilizado na atribuição da estrutura do discurso. A interação de uma série de outras características linguísticas e não linguísticas - sintáticas, pragmáticas, gestuais e elementos visuais - é o que faz com que a audiência infira a estrutura global de um determinado texto. Seria, portanto, interessante investigar a interação da prosódia com alguns desses elementos de contextualização no processo de demarcação da estrutura do discurso em Kisêdjê, como, por exemplo, a relação entre elementos prosódicos e pistas gestuais - algo que, aparentemente, eles exploraram com bastante regularidade. 


\section{Referências}

AOULADOMAR, F., 2005. A semantic analysis of instructional texts. IWCSO5. Tilburg.

AOULADOMAR, F., SAINT-DIZIER, P., 2005. Towards generating procedural texts: an exploration of their rhetorical and argumentative structure, $E W N L G$, Aberdeen.

ARIM, E., COSTA, F. \& FREITAS, T. 2003. A study on the reliability of two discourse segmentation models. Actas do VI Encontro para o Processamento Computacional da Língua Portuguesa Escrita e Falada (PROPOR 2003), Faro, Portugal.

BLAAUW, E. 1995. On the perceptual classification of spontaneous and read speech. Research Institute for Language and Speech, Utrecht University.

BOERSMA, P. 2005. Praat: doing phonetics by computer. http://www.fon.hum.uva.nl/praat/.

BROWN, G., CURRIE, K. \& KENWORTHY, J. 1980.

Questions of Intonation. London, Croom Helm.

CHAFE, W. 1989. Some things that narratives tell us about the mind. In B. B. A. Pellegrini. Narrative Thought and Narrative Language. Hillsdale, Lawrence Erlbaum.

CRUTTENDEN, A. 1997. Intonation. Cambridge, Cambridge University Press.

CRYSTAL, D. 1969. Prosodic Systems and Intonation in English. London, Cambridge University Press.

CUTLER, A., DAHAN, D. and DONSELAAR, W. 1994. Prosody in the comprehension of spoken language: A literature review. Language and Speech 40 (2):141-201 
GEE, J. P. \& GROSJEAN, F. 1984. Empirical evidence for narrative structure. Cognitive Science 8: 59-85.

GELUYKENS, R. \& SWERTS, M. 1994. Prosodic cues to discourse boundaries in experimental dialogues. Speech Communication 15: 69-77.

GROSZ, B. \& HIRSCHBERG, J. 1992. Some intonational characteristics of discourse structure. Proceeding of the International Conference on Spoken Language Processing, Banff.

GROSZ, B. J. and C. L. SIDNER. 1986. Attention, intention and the structure of discourse. Computational Linguistics 12(3): 175-204.

HAKODA, K. \& SATO, H. 1980. Prosodic rules in connected speech synthesis, Translation of the Institute of Electronics and Communication Engineers 63-D.

HENDERSON, A., GOLDMAN-EISLER, F. \& SKARBECK, A. 1965. Temporal patterns of cognitive activity and breath control in speech. Language and Speech 8: 236-242.

HIEKE, A. E., KOWAL, S. \& O'CONNELL, D. C. 1983. The trouble with "articulatory pauses". Language and Speech 26: 203-214.

HIRSCHBERG, J. \& GROSZ, B. 1992. Intonation features of local and global discourse structure. Proceeding of the DARPA Workshop on Spoken Language Systems, Arden House.

KOWAL, S. \& O'CONNELL, D. C. 1987. Some temporal aspects of stories told while or after watching a film. Bulletin of the Psychonomic Society 25(5): 364-366. 
LITMAN, D. J. \& PASSONNEAU, R. J. 1995. Combining Multiple Knowledge Sources for Discourse Segmentation. Proceeding of the Proceedings of the 33rd Annual Meeting of the Association for Computational Linguistics (ACL-95), Cambridge, MA.

MENN, L. \& BOYCE, S. 1982. Fundamental frequency and discourse structure. Language and Speech 25(4): 341-379.

NAKAJIMA, S. \& ALLEN, J. F. 1992. Prosody as a cue for discourse structure. Proceeding of the International Conference on Spoken Language Processing, Banff, Canada.

NAKATANI, C. H. \& HIRSCHBERG, J. 1995. Discourse structure in spoken language: Studies on speech corpora. Proceeding of the AAAI Symposium Series: Empirical Methods in Discourse Interpretation and Generation.

NOOTEBOOM, S. G. \& KRUYT, J. G. 1987. Accents, focus distribution, and the perceived distribution of given and new information: an experiment. Journal of the Acoustical Society of America 82(5): 1512-1524.

PASSONNEAU, R. J. \& LITMAN, D. J. 1993. Intentionbased segmentation: Human reliability and correlation with linguistic cues. Proceeding of the 31st Annual Meeting of the Association for Computational Linguistics (ACL-93), Columbus, Ohio.

PIJPER, J. R. and A. A. SANDERMAN. 1994. On the perceptual strength of prosodic boundaries and its relation to suprasegmental cues. Journal of the Acoustical Society of America 96 (4): 2037- 2047.

RODRIGUES, A. 1999. "Macro-Jê". In: Dixon, R.M.W.; Aikhenvald, A.Y. eds. The Amazonian Languages. Cambridge: Cambridge University Press. 
SWERTS, M. 1997. Prosodic features at discourse boundaries of different strenght. Journal of the Acoustical Society of America 101(1): 514-521.

VAN DONZEL, M. 1999. Prosodic Aspects of Information Structure in Discourse. Faculteit der Geesteswetenschappen. Amsterdam, University van Amsterdam: 195.

WICHMAN, A., HOUSE, J. \& RIETVELD, T. 1997. Peak displacement and topic structure. Proceeding of the ESCA Tutorial and Research Workshop on Intonation, Athens. 
